\title{
A model predictive algorithm for active control of nonlinear noise processes
}

\author{
Qi-Zhi Zhang ${ }^{\mathrm{a}, *}$, Woon-Seng Gan ${ }^{\mathrm{b}}$ and Ya-li Zhou ${ }^{\mathrm{a}}$ \\ ${ }^{a}$ Department of computer science and Automation, Beijing Institute of Machinery, P. O. Box 2865, Beijing, 100085, \\ People's Republic of China \\ ${ }^{\mathrm{b}}$ School of Electrical and Electronic Engineering, Nanyang Technological University, Singapore
}

Received 16 May 2004

Revised 30 August 2004

\begin{abstract}
In this paper, an improved nonlinear Active Noise Control (ANC) system is achieved by introducing an appropriate secondary source. For ANC system to be successfully implemented, the nonlinearity of the primary path and time delay of the secondary path must be overcome. A nonlinear Model Predictive Control (MPC) strategy is introduced to deal with the time delay in the secondary path and the nonlinearity in the primary path of the ANC system. An overall online modeling technique is utilized for online secondary path and primary path estimation. The secondary path is estimated using an adaptive FIR filter, and the primary path is estimated using a Neural Network (NN). The two models are connected in parallel with the two paths. In this system, the mutual disturbances between the operation of the nonlinear ANC controller and modeling of the secondary can be greatly reduced. The coefficients of the adaptive FIR filter and weight vector of NN are adjusted online. Computer simulations are carried out to compare the proposed nonlinear MPC method with the nonlinear Filter-x Least Mean Square (FXLMS) algorithm. The results showed that the convergence speed of the proposed nonlinear MPC algorithm is faster than that of nonlinear FXLMS algorithm. For testing the robust performance of the proposed nonlinear ANC system, the sudden changes in the secondary path and primary path of the ANC system are considered. Results indicated that the proposed nonlinear ANC system can rapidly track the sudden changes in the acoustic paths of the nonlinear ANC system, and ensure the adaptive algorithm stable when the nonlinear ANC system is time variable.
\end{abstract}

Keywords: Active noise control, model predictive control, nonlinear control, online modeling

\section{Introduction}

Noise reduction can be achieved by two different techniques. The first is using passive technique, which is based on the absorption and reflection properties of materials. Passive technique can present excellent results for high frequency noise, but its low frequency performance is poor. The other technique is using active noise control (ANC), which is based on the principle of wave interference. A secondary source is introduced to generate anti-noise of equal amplitude and opposite phase with reference to the primary noise. Active noise attenuation is an attractive method to achieve good low-frequency noise (below $1 \mathrm{kHz}$ ) reduction. A popular adaptive filtering algorithm used in the ANC is the filter-x LMS (FXLMS) algorithm in a finite impulse response (FIR) filter [1]. FXLMS is popular because of its simplicity, robustness and relatively low computational load. The block diagram of a single channel ANC system based on FXLMS algorithm is shown in Fig. 1. $x(k)$ is the primary noise, $e(k)$ is the error signal, $\boldsymbol{H}_{p}(z)$ is the primary path transfer function, and $\boldsymbol{w}(z)$ is the adaptive control filter. $u(k)$ is control signal, $y(k)$ is the secondary signal, and $\boldsymbol{H}_{m}(z)$ is the secondary path model. The FXLMS algorithm is an extension of the LMS

\footnotetext{
${ }^{*}$ Corresponding author. E-mail: zqzbim@yahoo.com.cn.
} 


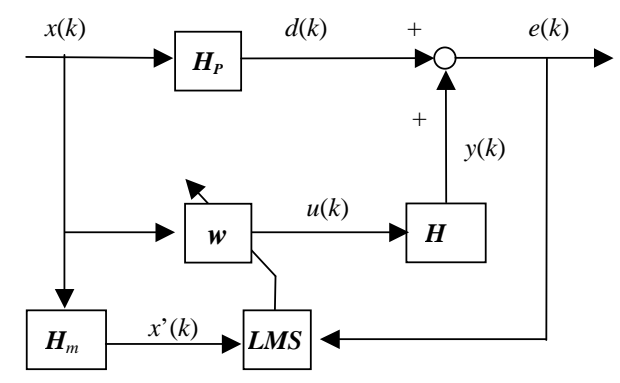

Fig. 1. Block diagram of an ANC system using FXLMS algorithm.

algorithm for ANC system, which takes into account of the influence of secondary path transfer function $\boldsymbol{H}(z)$ [2]. The secondary path $\boldsymbol{H}(z)$ comprises the D/A converter, smoothing filter, power amplifier, secondary loudspeaker, acoustic path from the loudspeaker to error microphone, error microphone, anti-aliasing filter, and A/D converter [3]. To design a practical ANC system, two important problems should be considered. Firstly, the secondary path may be time variable [3]. Secondly, the secondary path and primary path may exhibit nonlinear behaviors [4]. For a lightly damped mechanical system, the response changes of more than $6 \mathrm{~dB}$ in amplitude and $90^{\circ}$ in phase can occur near the natural frequency, because of a relatively small change in temperature [1]. If the phase difference for a particular frequency between the actual and the estimated model is more than $90^{\circ}$, the ANC system can become unstable [2]. Therefore, it is very important that on-line modeling of the secondary path is estimated fast and precisely for an ANC system [1-3].

The secondary path, $\boldsymbol{H}(z)$, may be estimated off-line prior to the operation of the ANC system. However, in some practical cases, $\boldsymbol{H}(z)$ can be time varying or nonlinear [5,6]. For these cases, on-line modeling of $\boldsymbol{H}(z)$ is required to ensure the convergence of the FXLMS algorithm for the ANC system. A number of methods for online modeling of the secondary path $\boldsymbol{H}(z)$ have been proposed [7,8]. These methods can be divided into two types [2, 3]. The first approach involves the injection of additional zero-mean white random noise into the ANC system, and utilizes a system identification method to estimate $\boldsymbol{H}(z)$. The auxiliary noise is uncorrelated with primary noise $x(k)$. The second approach, called the overall modeling algorithm, using control signal $u(k)$ to excite the secondary path model $\boldsymbol{H}_{m}(z)$, employs an extended least squares technique to model $\boldsymbol{H}(z)$. A detailed comparison of these two on-line modeling approaches has been reported in [7]. The main drawback of on-line modeling of $\boldsymbol{H}(z)$ is the mutual disturbances between the operation of the ANC controller and the modeling of the secondary path. However, more adaptive filters can be introduced to overcome this problem [3].

The multilayer perceptron neural network (NN) is used to control nonlinear plants [9]. The NN is a globalapproximated system, and the major problem in the NN-based ANC is its relatively slow learning (or convergence) process. To solve the problem mentioned above, several strategies can be adopted. One is the fast NN learning algorithm utilized in the control system [10], and the other is the NN enhanced controller used in the ANC system [11]. In addition, the local approximate neural network, radial basis function (RBF) networks [12] or fuzzy neural networks (FNN) [13], can be introduced to improve the performance of the convergence.

Recently, a model predictive control (MPC) technique has been introduced to the ANC system with on-line modeling techniques [14]. It can ensure the performances of adaptive control algorithm and the adaptive identification algorithm when both primary path and secondary path are linear and time-variable. In this paper, an MPC technique, using dynamic matrix control (DMC) is modified in the ANC system, whose primary path exhibits nonlinear behavior. An optimal technology is utilized to design the controller. NN is used to estimate the primary path and an FIR filter is used to estimate the secondary path $\boldsymbol{H}(z)$. An overall on-line modeling algorithm is adopted, and the NN and FIR filter are adaptively updated on-line by the extended LMS algorithm. This paper is organized as follows: Section 2 describes NN-based ANC system, and Section 3 describes the proposed DMC method for the nonlinear ANC system. Section 4 describes the adaptive DMC method with online the primary path and secondary path modeling. The NN-based FXLMS algorithm and the proposed nonlinear DMC algorithm are compared using computer simulations in Section 5. Finally, the conclusions are given in Section 6. 


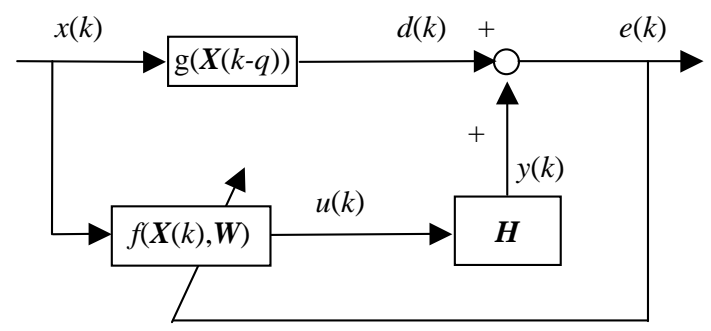

Fig. 2. Block diagram of a nonlinear control system.

\section{Adaptive neural networks for nonlinear ANC system}

An ANC system with nonlinear primary noise path is shown in Fig. 2. The secondary path is modeled with a FIR filter. The ANC system can be described by the following equation:

$$
e(k)=d(k)+y(k)=g(\boldsymbol{X}(k-q))+\sum_{j=0}^{N} h(j) u(k-j),
$$

where $\boldsymbol{X}(k)=[x(k) \quad x(k-1) \ldots x(k-n)]^{T}$ is the reference signal vector, $q$ is the time delay of the primary path, $u(k)$ is the output of nonlinear controller, and $h(j)(j=0,1 \ldots N)$ is the FIR filter coefficients of the secondary path model. If the time delay of the secondary path model is $d_{1}$, then $h(j)=0$ (for $j=0,1 \ldots d_{1}$ ). $d$ is the disturbance signal received at the error microphone, and $\mathrm{g}($.$) is a smoothing nonlinear function. The output of the \mathrm{NN}$ controller can be expressed as:

$$
u(k)=f(\boldsymbol{X}(k), \boldsymbol{W})
$$

A single hidden layer NN is selected as nonlinear controller, $\boldsymbol{W}^{I}$ is the link-weight vector between input layer and hidden layer and $\boldsymbol{W}^{O}$ is the link-weight vector between hidden layer and output layer. The output of the NN stated in [15], is given as:

$$
f(\boldsymbol{X}(k), \boldsymbol{W})=\sum_{i=1}^{L} \boldsymbol{W}_{i}^{O} \boldsymbol{Z}_{i}(k)=\boldsymbol{W}^{O} \boldsymbol{Z}(k)
$$

where

$$
Z_{i}(k)=f_{1}\left(\sum_{j=0}^{n} W_{j i}^{I} x(k-j)\right)=f_{1}\left(\boldsymbol{W}_{i}^{I} \boldsymbol{X}(k)\right),
$$

$f_{1}(x)=1 /\left(1+e^{-x}\right)$ is activation function of hidden neuron, and $\boldsymbol{W}=\left[\boldsymbol{W}^{I} \boldsymbol{W}^{O}\right]$ is the weight matrix. $L$ is the number of hidden neurons, $\boldsymbol{Z}(k)=\left[Z_{1}(k) Z_{2}(k) \ldots Z_{L}(k)\right]^{T}$ is the output vector of hidden neurons. The performance index can be described as:

$$
J(k)={ }_{2}^{1} e^{2}(k)={ }_{2}^{1}[y(k)+d(k)]^{2}
$$

The weight parameters can be adjusted according to the gradient descent method

$$
\boldsymbol{W}(k+1)=\boldsymbol{W}(k)-\mu \frac{\partial J(k)}{\partial \boldsymbol{W}(k)}=\boldsymbol{W}(k)-\mu e(k) \frac{\partial y(k)}{\partial \boldsymbol{W}(k)},,
$$

where $\mu$ is the learning rate. Applying the chain rule to Eq. (6):

$$
\begin{gathered}
\partial y(k) \\
\partial \boldsymbol{W}(k)
\end{gathered}=\sum_{j=0}^{N} \begin{gathered}
\partial y(k) \\
\partial u(k-j)
\end{gathered} \cdot \begin{gathered}
\partial u(k-j) \\
\partial \boldsymbol{W}(k)
\end{gathered}=\sum_{j=0}^{N} h(j) \cdot \begin{gathered}
\partial u(k-j) \\
\partial \boldsymbol{W}(k)
\end{gathered} .
$$

If the weights, $\boldsymbol{W}(k)$ are made to adapt slowly, the gradients of $u$ in Eq. (7) can be approximately written as: 


$$
\begin{gathered}
\partial u(k-j) \\
\partial \boldsymbol{W}(k)
\end{gathered} \approx \begin{gathered}
\partial u(k-j) \\
\partial \boldsymbol{W}(k-j)
\end{gathered}=\left.\begin{gathered}
\partial f(\boldsymbol{X}, \boldsymbol{W}) \\
\partial \boldsymbol{W}
\end{gathered}\right|_{\boldsymbol{X}=\boldsymbol{X}(k-j), \boldsymbol{W}=\boldsymbol{W}(k-j)}
$$

The gradient with respect to the weight $\boldsymbol{W}$ can be found in [15], as follows:

$$
\begin{aligned}
& \begin{array}{c}
\partial f(\boldsymbol{X}, \boldsymbol{W}) \\
\partial \boldsymbol{W}^{O}(k)
\end{array}=\boldsymbol{Z}(k)
\end{aligned}
$$

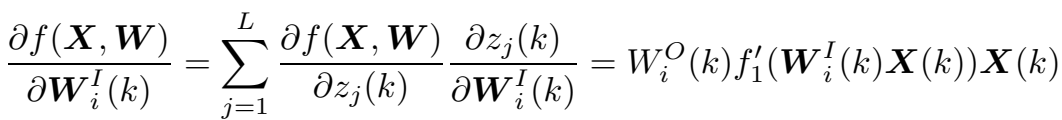

The weight $\boldsymbol{W}$ of NN controller can be adjusted online using the update rule Eq. (6) to Eq. (10).

\section{Dynamic matrix control (DMC) approach for nonlinear ANC system}

The time delay is a fundamental difficulty of process control. Consideration of this problem led to the development of model predictive control strategy [16]. The model predictive control is introduced in [17]. In the model predictive control algorithm, a dynamic model of plant is used to predict the effect of future actions of the control variables on the output. The future moves of the control variables are determined by optimization with the objective of minimizing the predicted error. Using the updated information (measurements) from the plant, the optimization [17] is repeated at each sampling time interval.

The DMC approach for linear ANC system [14] can be improved to treat the ANC system with nonlinear primary path (Fig. 2). Similar to [14], the performance objective is selected as follows:

$$
\begin{aligned}
& J(k)=\boldsymbol{e}^{T} \boldsymbol{e}+\Delta \boldsymbol{u}_{2}^{T} \mathbf{R} \Delta \boldsymbol{u}_{2}=(\boldsymbol{d}(k)+\hat{\boldsymbol{y}}(k))^{T}(\boldsymbol{d}(k)+\hat{\boldsymbol{y}}(k))+\Delta \boldsymbol{u}_{2}^{T} \boldsymbol{R} \Delta \boldsymbol{u}_{2} \\
& y(k)=\sum_{i=1}^{N} a_{i} \Delta u(k-i)+a_{N} u(k-N-1), \Delta u(k)=u(k)-u(k-1) \\
& d(k+j \mid k)=g(\boldsymbol{X}(k+j-q)), j=1,2, \ldots P \\
& \hat{\boldsymbol{y}}(k)=\boldsymbol{A} \Delta \boldsymbol{u}_{1}(k)+\boldsymbol{B} \Delta \boldsymbol{u}_{2}(k)+a_{s} \boldsymbol{u}(k) \\
& \hat{\boldsymbol{y}}(k)=[\hat{y}(k+1 \mid k), \hat{y}(k+2 \mid k), \cdots, \hat{y}(k+P \mid k)]_{P \times 1}^{T} \\
& \Delta \boldsymbol{u}_{1}(k)=[\Delta u(k-N+1), \Delta u(k-N+2), \cdots, \Delta u(k-1)]_{(N-1) \times 1}^{T} \\
& \Delta \boldsymbol{u}_{2}(k)=[\Delta u(k), \Delta u(k+1), \cdots, \Delta u(k+M-1)]_{M \times 1}^{T} \\
& \boldsymbol{u}(k)=[u(k-N), u(k-N+1), \cdots, u(k-N+P-1)]_{P \times 1}^{T} \\
& A=\left[\begin{array}{ccccc}
a_{N} & a_{N-1} & \ldots & \ldots & a_{2} \\
& a_{N} & & \ldots & a_{3} \\
& & \ddots & \vdots & \vdots \\
& & a_{N} & \cdots & a_{P+1}
\end{array}\right], \boldsymbol{B}=\left[\begin{array}{cccc}
a_{1} & & & \\
a_{2} & a_{1} & & \\
& & \ddots & \\
& & & a_{1} \\
& \vdots & \vdots & \vdots \\
a_{P} & a_{P-1} & \cdots & a_{P-M+1}
\end{array}\right] \\
& \boldsymbol{d}(k)=[d(k+1 \mid k), d(k+2 \mid k), \cdots, d(k+P \mid k)]_{P \times 1}^{T}
\end{aligned}
$$


where $d(k+j \mid k)=$ predicted value of $d$ at time $k+j$ based on information available at time $k$

$\hat{y}(k+j \mid k)=$ modeling predicted value of $y$ at time $k+j$ based on information available at time $k$

$a_{i}=$ model step response coefficient of secondary path

$a_{s}=$ stable value of step response coefficient of secondary path

$N=$ truncation order of secondary path

$n=$ truncation order of primary path

$P=$ horizon length

$d_{1}=$ the time delay of the secondary path

$q=$ the time delay of the primary path

$M=$ number of control variable moves in the future $(\Delta u(k+j)=0$, when $j>M ; M<P)$

$\boldsymbol{R}=$ weighting matrix

$\boldsymbol{A}, \boldsymbol{B}=$ Dynamic Matrices

At time $k, \boldsymbol{u}(k)$ and $\Delta \boldsymbol{u}_{1}(k)$ are the available information. The $M$-step ahead incremental control variable moves vector, $\Delta \boldsymbol{u}_{2}(k)$, are selected to minimize the quadratic objective

$$
\Delta \boldsymbol{u}_{2}(k)=-\left[\boldsymbol{B}^{T} \boldsymbol{B}+\boldsymbol{R}\right]^{-1} \boldsymbol{B}^{T}\left[\boldsymbol{d}(k)+\boldsymbol{A} \Delta \boldsymbol{u}_{1}(k)+a_{s} \boldsymbol{u}(k)\right]
$$

at time $k$. Only the first coefficient of $\Delta \boldsymbol{u}_{2}(k)$ is required to be computed, and the control variable $u(k)=$ $u(k-1)+\Delta u(k)$ is implemented. At time $k+1$, the computation is repeated with an increment of one time interval. From Eq. (13), one can find that the horizon length must be chosen as $P \leqslant q$, since at time $k$, the predicted value of $d$ at time $k+P$ has not be obtained. In addition, the horizon length should be $d_{1}<P$, where $d_{1}$ is the time delay of the secondary path.

If the models are accurate, and there are no additive disturbances in the ANC system, the error $e(k)$ can be manipulated to zero. However modeling error and additive disturbances always exist in the practical control systems. The predicted values must be corrected by the measured error feedback:

$$
\hat{\boldsymbol{y}}_{1}(k+1)=\hat{\boldsymbol{y}}(k+1)+\boldsymbol{c} e(k+1)
$$

where $\boldsymbol{c}$ is a correcting vector. After error feedback correction, the $M$-step ahead incremental control variable moves vector, $\Delta \boldsymbol{u}_{2}(k)$, becomes

$$
\Delta \boldsymbol{u}_{2}(k)=-\left[\boldsymbol{B}^{T} \boldsymbol{B}+\boldsymbol{R}\right]^{-1} \boldsymbol{B}^{T}\left[\boldsymbol{d}(k)+\boldsymbol{A} \Delta \boldsymbol{u}_{1}(k)+a_{s} \boldsymbol{u}(k)+\boldsymbol{c} e(k)\right]
$$

\section{Adaptive DMC approach for nonlinear ANC system}

In the last section, the dynamic matrix control (DMC) algorithm for nonlinear ANC system is presented. The primary path and the secondary path are estimated off-line prior to the operation of the ANC system. For the secondary path off-line modeling, a zero-mean white random noise excitation signal can be generated by a computer. But the same excitation signal is difficult to obtain for the primary path modeling, because the reference signal sampled by the computer has passed through the reference microphone, anti-aliasing filter, and A/D converter. The precision of primary path modeling would be also degraded by the nonlinear influence. So the online modeling technique should be adopted to improve the performance of the nonlinear ANC system.

Figure 3 gives the block diagram of the adaptive ANC system with online nonlinear primary path modeling, and the secondary path model has been estimated off-line. In Fig. 3, the $\boldsymbol{H}_{m}$ is the estimated impulse responses of the secondary path and $f(\boldsymbol{X}(k-q), \boldsymbol{W})$ is the neural network model of nonlinear primary path. The coefficient vector of the NN can be updated as follows

$$
\boldsymbol{W}(k+1)=\boldsymbol{W}(k)+\mu e_{p}(k) \begin{gathered}
\partial f(\boldsymbol{X}(k-q), \boldsymbol{W}), \\
\partial \boldsymbol{W}
\end{gathered}
$$

where $\mu$ is the learning rate. The gradients in Eq. (24) can be found in Eqs (9) and (10). Comparing Eq. (24) with Eq. (6) to Eq. (10), one can find that the weight adaptive processes (in Eq. (24)) is independent with the secondary 


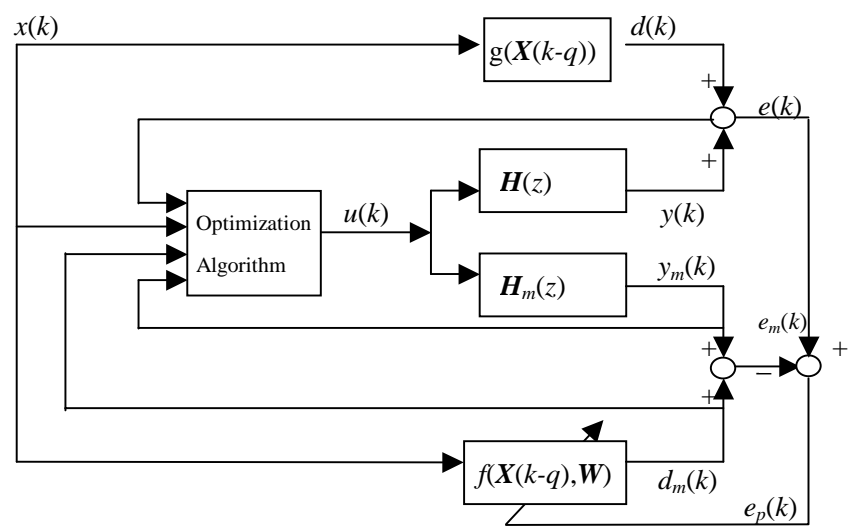

Fig. 3. Block diagram of adaptive ANC system with online primary path modeling.

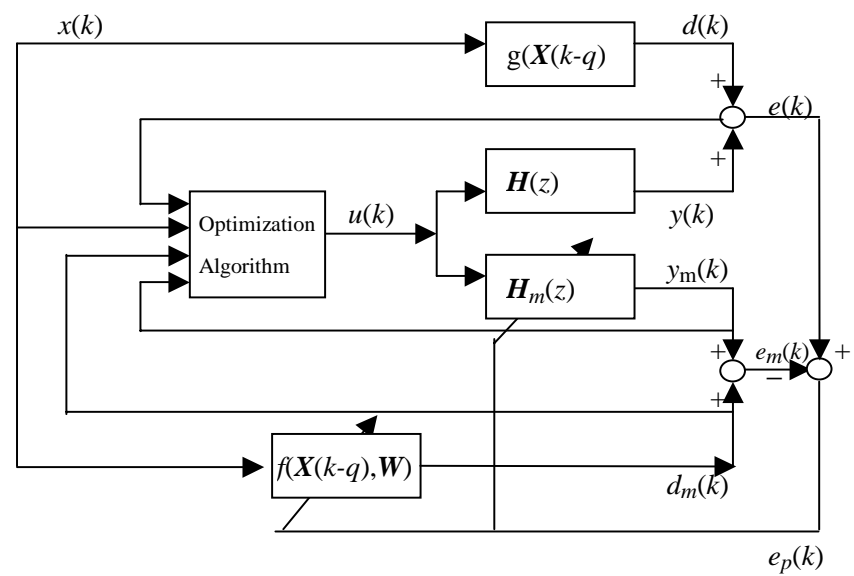

Fig. 4. Block diagram of adaptive ANC system with online secondary path modeling.

path. Therefore, the secondary path does not degrade the convergence performance of the primary path modeling. The nonlinear adaptive control ANC system runs as follows:

(1) At time $k$, the first coefficient of incremental control variable moves vector, $\Delta \boldsymbol{u}_{2}(k)$, is computed, and the instantaneous control variable $u(k)=u(k-1)+\Delta u(k)$ is implemented. From Eq. (11) to Eq. (23), the impulse responses of the secondary path $\boldsymbol{H}(\mathrm{z})$ and nonlinear primary path $\mathrm{g}(\boldsymbol{X}(k-q))$, are replaced by the estimated impulse responses of the secondary path $\boldsymbol{H}_{m}(z)$ and NN model of nonlinear primary path $f(\boldsymbol{X}(k-q), \boldsymbol{W})$, respectively.

(2) The weight vector of NN model $f(\boldsymbol{X}(k-q), \boldsymbol{W})$ is updated by Eq. (24).

(3) At time $k+1$, the computation is repeated with an increased of one time interval.

In the adaptive control algorithm, the dynamic matrices $\boldsymbol{A}, \boldsymbol{B}$ and the inverse matrix in Eq. (23) can be computed off-line before the operation of the nonlinear ANC system since they are invariable when the adaptive nonlinear ANC system is running. The computing load of the adaptive algorithm is similar to that of the NN-based FXLMS algorithm when the nonlinear ANC system with DMC algorithm is running. When $\boldsymbol{H}(z)$ is time varying or $x(k)$ exists, the on-line secondary modeling algorithm must be selected to ensure the convergence of the adaptive nonlinear ANC system. A model predictive algorithm for nonlinear ANC system with online secondary path modeling is proposed. The block diagram of proposed nonlinear DMC algorithm is shown in Fig. 4.

This on-line modeling algorithm is similar to overall modeling algorithm. The impulse response coefficient vectors of the secondary path model and the weight vectors of NN model can be updated as follows:

$$
\boldsymbol{H}_{m}(k+1)=\boldsymbol{H}_{m}(k)+\eta e_{p}(k) \boldsymbol{u}(k)
$$




$$
\boldsymbol{W}(k+1)=\boldsymbol{W}(k)+\mu e_{p}(k) \begin{gathered}
\partial f(\boldsymbol{X}(k-q), \boldsymbol{W}) \\
\partial \boldsymbol{W}
\end{gathered}
$$

where $\mu$ and $\eta$ is the learning rates, $\boldsymbol{u}(k)=\left[\begin{array}{ll}u(k) & u(k-1) \ldots u(k-N)\end{array}\right]$. At first glance, the model predictive algorithm for nonlinear ANC system with on-line secondary path modeling is similar to the previous adaptive algorithm. But they are different in essence. The later algorithm is more complex than the other algorithm when the adaptive nonlinear ANC system is running. The adaptive control ANC system with online secondary path modeling runs as follows:

(1) At time $k$, the dynamic matrices $\boldsymbol{A}, \boldsymbol{B}$ and the inverse matrix in Eq. (23) is computed, and the first coefficient of incremental control variable moves vector, $\Delta \boldsymbol{u}_{2}(k)$, is computed. After that, the instantaneous control variable $u(k)=u(k-1)+\Delta u(k)$ is implemented. In Eq. (11) to (23), the impulse responses of the secondary path $\boldsymbol{H}$ and nonlinear primary path $g(\boldsymbol{X}(k-q))$, are replaced by the estimated impulse responses of the secondary path $\boldsymbol{H}_{m}(z)$ and NN model of nonlinear primary path $f(\boldsymbol{X}(k-q), \boldsymbol{W})$ respectively. The impulse response coefficient vectors of secondary path model and weight vectors of NN model of primary path are updated by Eq (25) and (26).

(2) At time $k+1$ the computation is repeated with the horizon moved by one time interval.

Unlike former algorithm, the dynamic matrices $\boldsymbol{A}, \boldsymbol{B}$ and the inverse matrix in Eq. (23) are computed on-line and iteratively with the operation of the ANC system in this adaptive control algorithm. The computing load of the adaptive algorithm is heavier than that of the previous algorithm, especially when the number of control variable moves in the future, $M$, is large. This is the cost of having on-line secondary path modeling.

\section{Computer simulations}

In this section, some illustrative results are presented to compare the performances of the neural network based nonlinear ANC systems and the DMC based nonlinear ANC system. A personal computer with Pentium $1.8 \mathrm{GHz}$ processor and a DRAM of $256 \mathrm{MB}$ is used to implement the simulations. The sampling frequency used for this simulation is $1,000 \mathrm{~Hz}$. The disturbance signal is chosen to be a $100 \mathrm{~Hz}$ pure tone signal plus a Gaussian white noise signal, which can characterize a general noise model. For example, this model can characterize the fan noise. The rotation of fan generates tone signal, and the turbulence flow generates white noise signal. The adaptive filter model of secondary path, $\boldsymbol{H}_{m}(z)$, is selected as a 4-taps FIR filter, and the NN architecture used is 3-2-1. The models in the NN-based FXLMS algorithm are the same as the model in the DMC based algorithm. A nonlinear active noise control example is selected, and the responses of the acoustic path, which are similar as in [4], are chosen as follows:

The primary acoustic path is selected as

$$
d(k)=x(k-4)-0.3 x(k-5)+0.2 x(k-6)+0.8 x^{2}(k-5) .
$$

The secondary acoustic path from secondary source to error microphone is

$$
y(k)=u(k-1)+1.5 u(k-2)-u(k-3) .
$$

The secondary path has a zero at $z=-2$, which is outside the unit circle and a 1-sample delay is used. which resulted in a nonminimum-phase system. It contains the main characteristics of the real secondary path. Computer simulations are carried out to compare the performance of proposed the DMC based nonlinear ANC system and the neural network based nonlinear FXLMS ANC systems. The simulations using the proposed nonlinear DMC method are tested on three cases, and the results are compared with the results using nonlinear FXLMS algorithm.

Case 1: both primary path and secondary path are time invariable. The secondary model, $\boldsymbol{H}_{m}(\mathrm{z})$, is estimated using off-line modeling technique as follows:

$$
\boldsymbol{H}_{m}(z)=z^{-1}+1.2 z^{-2}-z^{-3}
$$

The NN-based nonlinear FXLMS algorithm for ANC system is computed first. The initial values of adaptive weight matrix $\boldsymbol{W}$ are selected as small random numbers. The step size for updating $\boldsymbol{W}$ is $\mu=0.01$. This is compared to the DMC algorithm for ANC system with on-line primary path modeling. The parameters used in these algorithms are selected as follows: The truncation order of the secondary path is $N=4$, the truncation order of primary path is $n=3$, the horizon length is $P=4$, the number of control variable moves in the future is $M=2$, 

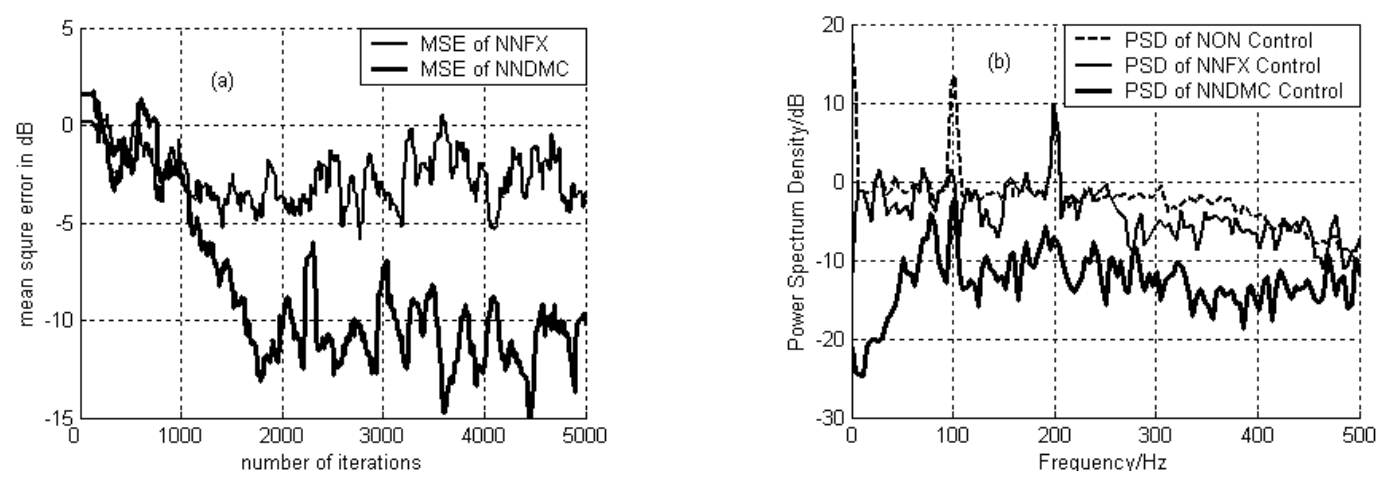

Fig. 5. (a) The error curves of proposed nonlinear DMC algorithm and NN-based nonlinear FXLMS algorithm for ANC system with off-line secondary path modeling. (b) Power spectrum of active noise canceling errors for ANC system with off-line secondary path modeling. Results from the NN-based controller are indicated in thin line, and thick line is used for the nonlinear DMC controller; the dished line indicates the results when ANC is turned off.

and weighting matrix is $\boldsymbol{R}=0.06 * \boldsymbol{I}$, where $\boldsymbol{I}$ is identical matrix. All the elements of the correcting vector $\boldsymbol{c}$ are chosen as 0.1 . The initial value of adaptive weight matrix $\boldsymbol{W}$ is selected as small random numbers. The step size for updating $\boldsymbol{W}$ is $\mu=0.125$. Figure 5(a) shows the mean square error curves versus the iterations (samples), and Fig. 5(b) shows the simulating results of the canceling errors between 4,000th to 5,000th iterations in the frequency domain. The result of DMC for nonlinear ANC system with on-line primary path modeling is shown in solid thick threads, and the result of NN-based nonlinear FXLMS is shown in solid thin lines. From the results shown in Fig. 5(a), it can be seen that after 2,000 iterations, the mean square errors of proposed nonlinear DMC method settle on the level of $-12 \mathrm{~dB}$, and that of NN-based method settle on the level of $-3 \mathrm{~dB}$. The nonlinear DMC method can obtain about $10 \mathrm{~dB}$ additional noise decrease compared with the NN-based method. There is a $200 \mathrm{~Hz}$ peak in the noise spectrum, which is produced by the square nonlinearity in the primary acoustic path. The NN-based nonlinear ANC system can reduce the $100 \mathrm{~Hz}$ noise by $10 \mathrm{~dB}$, but it cannot reduce the $200 \mathrm{~Hz}$ tone and the broadband noise. The proposed DMC based nonlinear ANC system can reduce the $100 \mathrm{~Hz}$ noise by $15 \mathrm{~dB}$, and can also reduce the $200 \mathrm{~Hz}$ noise by $18 \mathrm{~dB}$. The broadband noise can be reduced by about $10 \mathrm{~dB}$. From Fig. 5(a) and (b), one can find that the convergence rates of proposed DMC based method are much faster than that of the NN-based algorithm. The performance of DMC based nonlinear ANC system is superior to that of NN-based ANC system.

Case 2: The secondary path is obtained using off-line modeling technology as in case 1, but the primary path has a sudden change at the 2,000th iteration. The linear part of the primary path's impulse response changes to its opposite value, and nonlinear part remains unchanged.

$$
d(k)=-x(k-4)+0.3 x(k-5)-0.2 x(k-6)+0.8 x^{2}(k-5)
$$

The step size for updating weight vector $\boldsymbol{W}$ in the DMC based nonlinear ANC system is $\mu=0.1$. The other parameters are chosen as in case 1.

Figure 6(a) shows the mean square error curves of both DMC and nonlinear FXLMS. The solid thick lines indicate results of proposed nonlinear DMC algorithm, and the solid thin lines indicate result of nonlinear FXLMS algorithm. From the results shown in Fig. 6(a), it can be seen that after about 300 iterations, the mean square errors of proposed nonlinear DMC method settled to the same level as the errors before the sudden change of primary path. For NN-based nonlinear ANC system, more iterations(about 2000 iterations) are required to settle to the error level before the sudden change of primary path. In addition, the proposed nonlinear DMC method can track the change of the primary path very fast. Figure 6(b) shows the simulation results of the canceling errors between the 4,000th to 5,000 th iterations in the frequency domain. Similar to case 1, the NN-based nonlinear ANC system can only cancel the main noise $(100 \mathrm{~Hz})$. The DMC based nonlinear ANC system can cancel the main noise $(100 \mathrm{~Hz})$, the noise $(200 \mathrm{~Hz})$ produced by the square nonlinear in the primary acoustic path, and the broadband noise.

Case 3: The secondary path is obtained using on-line modeling technology. The secondary path experiences sudden change after 2000 iteration, the coefficients of impulse response of the secondary path change to its opposite values, 

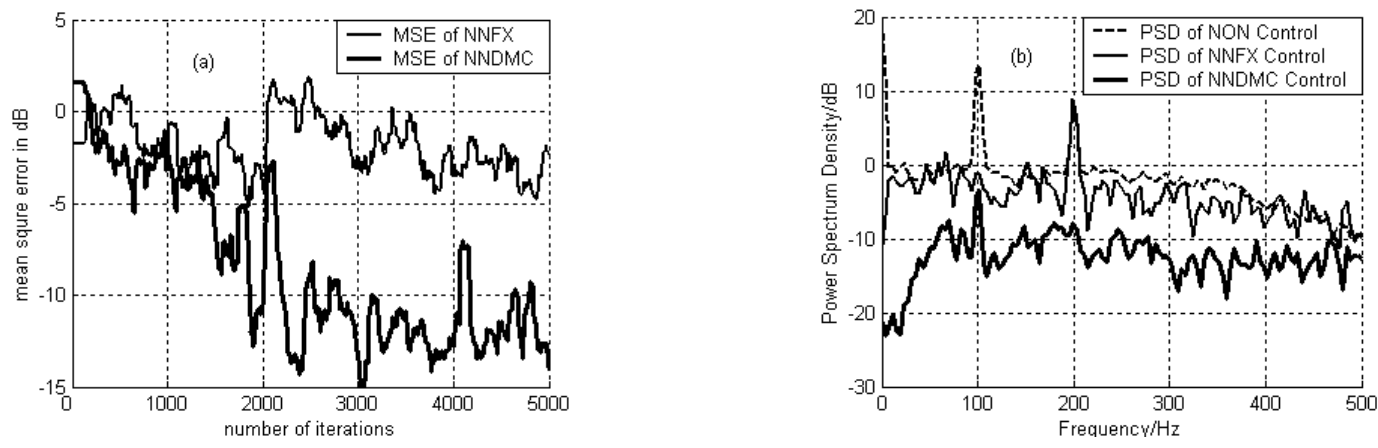

Fig. 6. (a) The error curves of proposed nonlinear DMC algorithm and NN-based nonlinear FXLMS algorithm for ANC system with off-line secondary path modeling. (b) Power spectrum of active noise canceling errors for ANC system with off-line secondary path modeling. The thin line shows the results from NN-based controller, the thick line shows the results from nonlinear DMC controller, and the dished line shows the results when ANC is turned off.
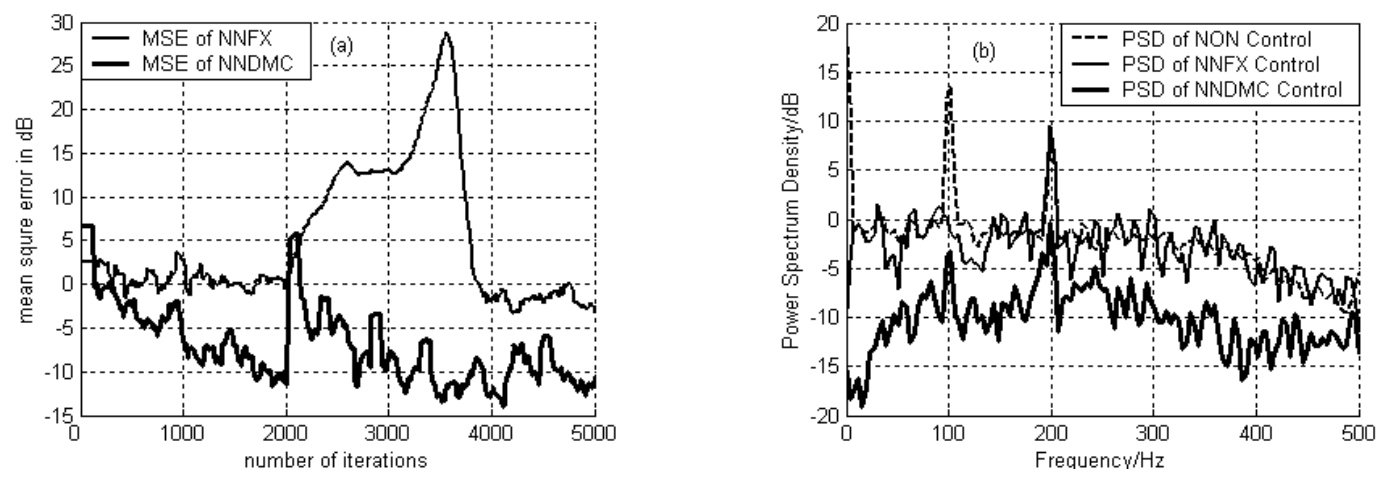

Fig. 7. (a) The error curves of proposed nonlinear DMC algorithm and NN-based nonlinear FXLMS algorithm for ANC system with on-line secondary path modeling. (b) Power spectrum of active noise canceling errors for ANC system with on-line secondary path modeling. The thin line for NN-based controller, the thick line for nonlinear DMC controller, and the dished line for ANC turn off.

$$
y(k)=-u(k-1)-1.5 u(k-2)+u(k-3) .
$$

The initial values of secondary model, $\boldsymbol{H}_{m}(\mathrm{z})$, is chosen as follows:

$$
\boldsymbol{H}_{m}(z)=0.1 z^{-1}+0.2 z^{-2}-0.1 z^{-3}
$$

The step size for updating the weight vector $\boldsymbol{W}$ of NN is $\mu=0.004$ in nonlinear FXLMS based ANC system. The step size for updating the weight vector $\boldsymbol{W}$ of NN is $\mu=0.1$ in nonlinear DMC based ANC system. The other parameters are chosen similar to case 1.

Figure 7(a) and (b) show the mean square error curves and the simulating results of the canceling errors between 4000th to 5000th iterations in the frequency domain. From the results shown in Fig. 7(a), it can be seen that after about 600 iterations, the mean square errors of proposed nonlinear DMC method settle to about same error level as before the sudden change of secondary path. For NN-based nonlinear ANC system, about 2000 iterations are required to settle on the level as the errors before the sudden change of secondary path. Figure 8 shows the time histories of the signals in the last 50 iterations. The secondary signal is of almost equal amplitude and opposite phase with reference to the primary noise. It can be found that the change of secondary path has more influence on the performance of the nonlinear ANC systems than the change of primary path. The proposed nonlinear DMC method can track the change of the secondary path very fast. But the NN-based ANC system has a long transition times, and the error shot up to about $30 \mathrm{~dB}$ during the transition period. In practice, large error would make the data converters and transducers saturate, which in turns saturates the adaptive system [1]. 


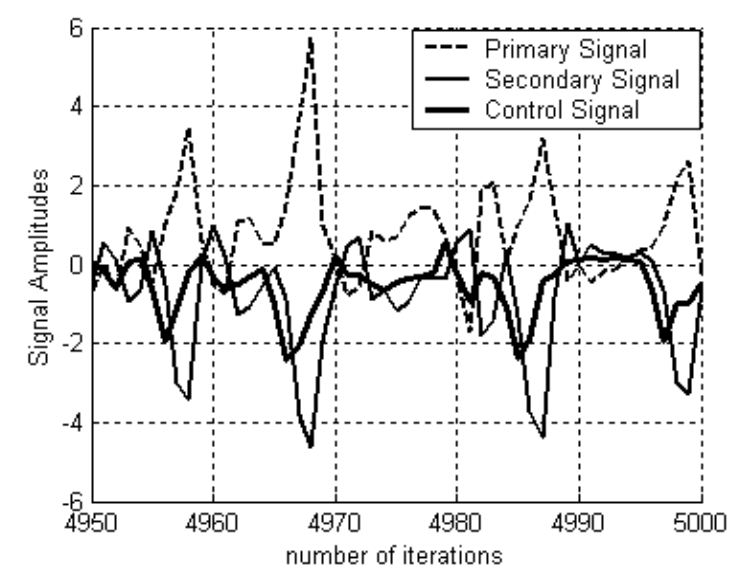

Fig. 8. Time histories of the signals in the last 50 iterations. The dashed line shows the primary signal, the thin line shows the secondary signal, and the thick line shows the control signal.

\section{Conclusions}

The designing of the secondary path is the key step for constructing an active noise control (ANC) system. The time delay and time variable are the inherent characteristics of the secondary path. The model predictive control utilizes explicit identifiable model to predict the responses of the plant, so that the increment of the control can be computed by the predictive errors. The DMC uses the step responses of the plant, which are easily obtained in practice. A modified nonlinear DMC algorithm for the ANC system is proposed in this paper. It can deal with the nonlinear of the primary path, the time delay and time variable of the secondary path. Since the adaptive filter for modeling the secondary path and the NN for modeling the primary path are collaterally arranged, the mutual disturbances between the operation of the nonlinear ANC controller and modeling of the secondary can be greatly reduced. Computer simulations have been presented, which showed that the proposed nonlinear DMC method is very effective to deal with the nonlinearity in the primary path, the time delay and time variable in the secondary path of the ANC system. It can rapidly track the sudden changes in the secondary path and the primary path of the ANC system. In addition, the proposed nonlinear DMC algorithm is robust to the sudden changes in the acoustic paths. The paper does not address the effect of actuator feedback on the reference sensor. The effect must be considered in a practical ANC system when the reference sensor is close to the actuator.

\section{Acknowledgments}

This research is supported by Science Founds of Educational Committee of Beijing City (KM200311232137) and Training Funds for Elitist of Beijing

\section{References}

[1] S.J. Elliott, Signal processing for active control, Academic Press, 2001.

[2] X.J. Qiu and C.H. Hansen, A modified filtered-x LMS Algorithm for active control of periodic noise with on-line cancellation path modeling, Journal of Low Frequency Noise, Vibration and Active Control 19 (2000), 35-46.

[3] M. Zhang, H. Lan and W. Ser, Cross-updated active noise control system with online secondary path modeling, IEEE Trans on Speech and Audio Processing 9 (2001), 598-602.

[4] L. Tan and J. Jiang, Adaptive volterra filters for active control of nonlinear noise processes, IEEE Trans. On Signal Processing 49 (2001), $1667-1676$.

[5] S.J. Elliott, Active control of nonlinear systems, Noise Control Engineering 49 (2001), 30-53.

[6] L.J. Erksson and M.A. Allie, Use of random noise for online transducer estimate in an adaptive attenuation system, Journal of the Acoustical Society of America 85 (1989), 797-802. 
[7] C. Bao, P. Sas and B.H. Van, Comparison of two online identification algorithms for active noise control, In Proc. Resent Advances in Active Control of Sound and Vibration, Technomic, Lancaster, PA, 1993, pp. 38-51.

[8] S.M. Kuo and D.R. Morgan, Active noise control: a tutorial review, IEEE Proceedings 87 (1999), $973-993$.

[9] M. Bouchard, B. Pailard and C.T.L. Dinh, Improved training of neural networks for the nonlinear active control of sound and vibration, IEEE Trans. on Neural Networks 10 (1999), 391-401.

[10] M. Bouchard, New recursive-least-squares algorithms for non-linear active control of sound and vibrations using neural networks, IEEE Trans. on Neural Networks 12 (2001), 135-147.

[11] Q.Z. Zhang and Y.L. Jia, Active Noise hybrid feedforward /feedback Control Using Neural Network Compensation, ASME J. of Vibration And Acoustics 124 (2002), 100-104.

[12] P. Strauch and B. Mulgrew, Active control of nonlinear noise processes in a linear duct, IEEE Trans. On Signal Processing 46 (1998), 2404-2412.

[13] Q.Z. Zhang and W.S. Gan, Active noise control using a simplified fuzzy neural network, Journal of sound and vibration 272 (2004), 437-449.

[14] Q.Z. Zhang and W.S. Gan, A model predictive algorithm for active noise control with online secondary path modelling, Journal of sound and vibration 270 (2004), 1056-1066.

[15] Q.Z. Zhang and Y.L. Jia, Active noise feedback control using a neural network, Shock and Vibration 8 (2001), 15-19.

[16] B.G. Joël and V. Cyril, Robust multivariable predictive control, IEEE Control Systems Magazine 21 (2001), 54-65.

[17] E.G. Carlos, M.P. David and M. Manfred, Model predictive control: theory and practice - a survey, Automatica 25 (1989), $335-348$. 

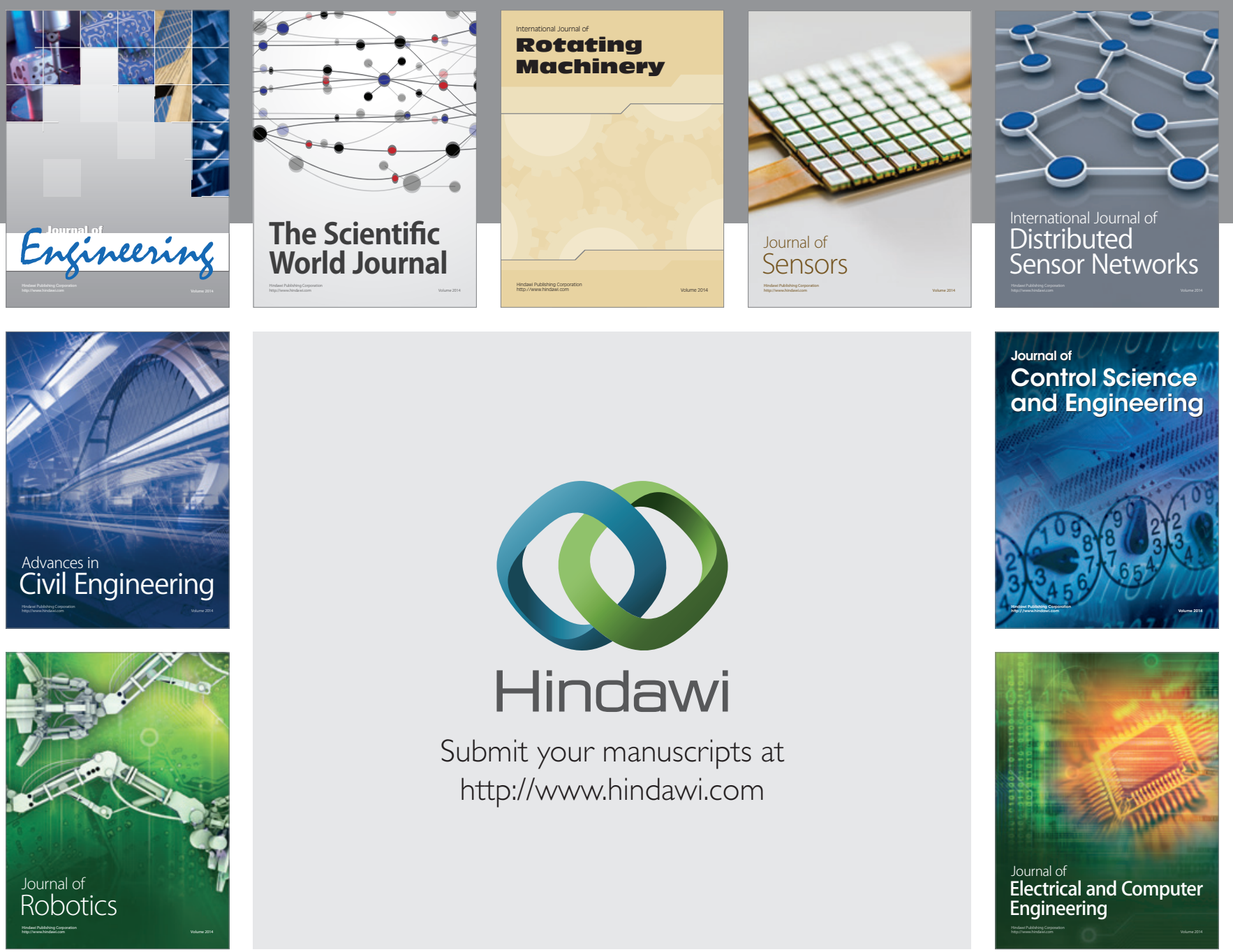

Submit your manuscripts at

http://www.hindawi.com
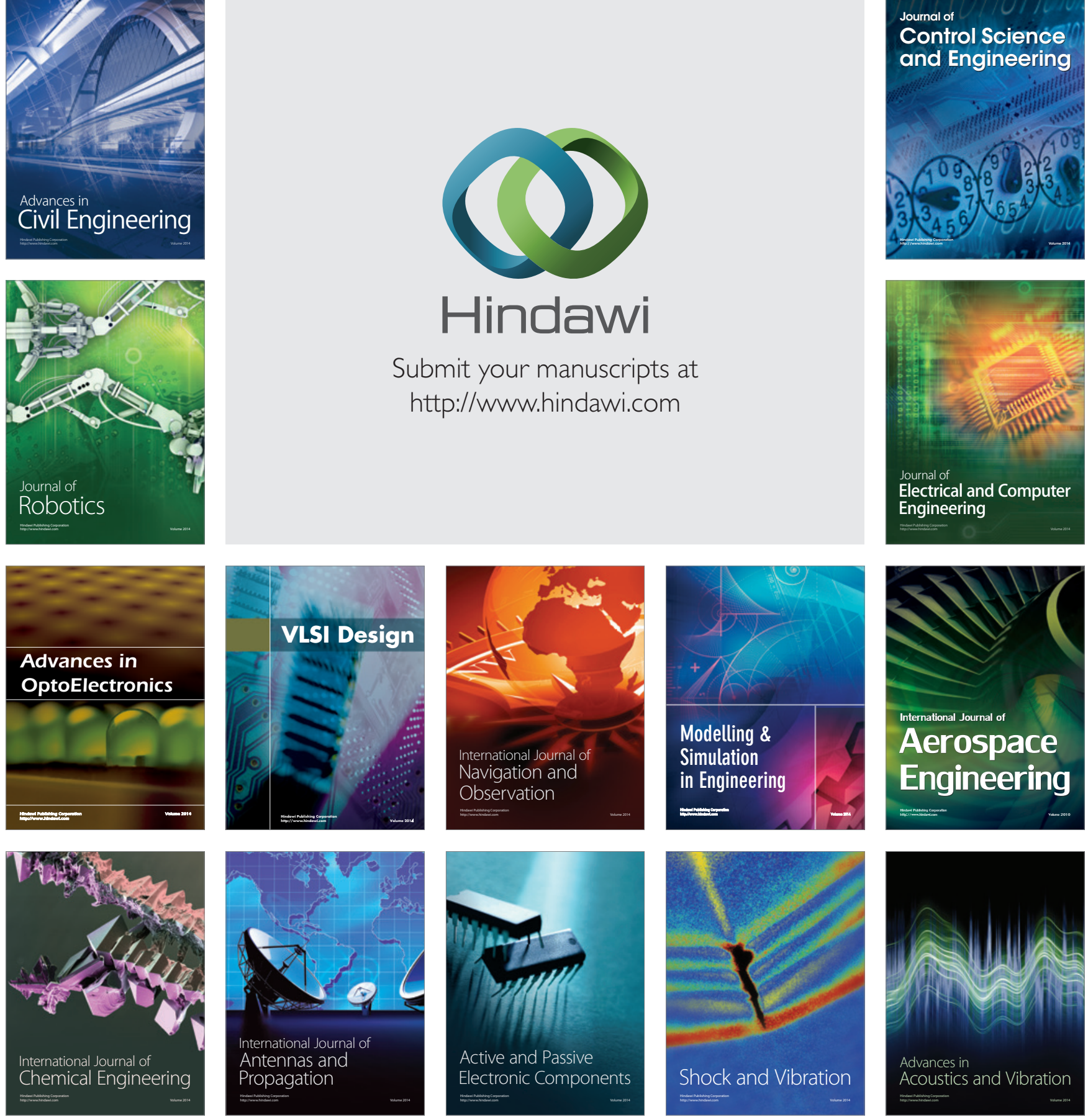